\title{
PARTITION ALGEBRAS FOR ALMOST-DISJOINT FAMILIES
}

BY

\author{
JAMES E. BAUMGARTNER ${ }^{1}$ AND MARTIN WEESE
}

\begin{abstract}
A set $a \subseteq \omega$ is a partitioner of a maximal almost-disjoint faculty $F$ of subsets of $\omega$ if every element of $F$ is almost contained in or almost-disjoint from $a$. The partition algebra of $F$ is the quotient of the Boolean algebra of partitioners modulo the ideal generated by $F$ and the finite sets. We show that every countable algebra is a partition algebra, and that $\mathrm{CH}$ implies every algebra of cardinality $\leqslant 2^{\boldsymbol{\aleph}_{0}}$ is a partition algebra. We also obtain consistency and independence results about the representability of Boolean algebras as partition algebras.
\end{abstract}

0. Introduction. Two subsets $a$ and $b$ of $\omega$ are almost-disjoint if $a \cap b$ is finite. An almost-disjoint family, for the purposes of this paper, is an infinite set of infinite, pairwise almost-disjoint subsets of $\omega$. We shall be interested in maximal almost-disjoint families (called mad families by Mathias in [7]), which in particular must be uncountable.

Let $F$ be a mad family. A set $a \subseteq \omega$ is a partitioner of $F$ iff $\forall b \in F$ either $b-a$ or $b \cap a$ is finite. The set of partitioners of $F$ forms a Boolean algebra $B$. A partitioner of $F$ is nontrivial if it does not belong to the ideal $I$ in $B$ generated by $F$ together with the finite sets. The algebra $B / I$ is called the partition algebra of $F$. If a Boolean algebra is isomorphic to the partition algebra of some mad family then we say that the algebra is representable.

It is our hope that this notion of partition algebra, due to the second author, will provide a useful way to classify almost-disjoint families. Other methods of classification have been studied in [ 3 and $\mathbf{9}$ ].

We are primarily concerned with determining when a given Boolean algebra is representable. We begin in $\$ 1$ with some technical results which yield, in $\S 2$, the fact that every countable Boolean algebra is representable, and that certain uncountable algebras are always representable as well. We also show here that if $B$ is a Boolean algebra and $|B|$ is smaller than the Rothberger cardinal $p$, then $B$ is representable. Hence, in particular, Martin's Axiom implies that every Boolean algebra of cardinality $<2^{\aleph_{0}}$ is representable. We show in $\S 3$ that the continuum hypothesis $(\mathrm{CH})$ implies that every Boolean algebra of cardinality $\leqslant 2^{\aleph_{0}}$ is representable, and in $\S 4$ we show it consistent that $\mathrm{CH}$ fails and no algebra containing the free algebra on $\boldsymbol{\aleph}_{2}$ generators is representable. Hence, in particular, it is consistent that no infinite

Received by the editors April 29, 1981.

1980 Mathematics Subject Classification. Primary 03E50; Secondary 03E05, 06E05.

Key words and phrases. Boolean algebra, almost-disjoint, Martin's Axiom, Rothberger property, continuum hypothesis, forcing and generic sets.

${ }^{1}$ Research partially supported by National Science Foundation grant number MCS7903376. 
complete Boolean algebra is representable. The paper concludes in $\S 5$ with a list of open problems.

Our set-theoretical notation is standard. $P(X)$ denotes the power-set of $X$, and $P(\omega)$ is generally regarded as a Boolean algebra with the usual set-theoretic operations. The meet, join and complement operations in an abstract Boolean algebra are denoted by $\wedge, \vee$ and ${ }^{-}$, but for subalgebras of $P(\omega)$ the set-theoretic operations are used. Throughout the paper, Fin denotes the set of finite subsets of $\omega$. We will be concerned with algebras embeddable in $P(\omega) /$ Fin. It is well known that every algebra of cardinality $\leqslant \boldsymbol{\aleph}_{1}$ is embeddable in $P(\omega) /$ Fin. See [2, Theorem 14.12].

We denote $\{f: f$ maps $a$ into 2$\}$ by ${ }^{a} 2$.

If $(P, \leqslant)$ is a partial ordering and $D \subseteq P$ then $D$ is dense in $P$ iff $\forall p \in P \exists q \in$ $D q \leqslant p$. Given a family $\left\{D_{i}: i \in I\right\}$ of dense subsets of $P$, we say that $G \subseteq P$ is generic with respect to $\left\{D_{i}: i \in I\right\}$ iff:

(1) $\forall p, q \in G \exists r \in G r \leqslant p, q$;

(2) $\forall p \in G \forall q \geqslant p q \in G$;

(3) $\forall i \in I G \cap D_{i} \neq 0$.

All the mad families we construct will have cardinality $2^{\aleph_{0}}$. Hechler [4] has shown it consistent that mad families of cardinality $<2^{\aleph_{0}}$ exist, but such families lead to a special class of Boolean algebras, the superatomic algebras, which we do not consider here, except for a brief treatment of finite-cofinite algebras.

THEOREM 0.1. Suppose $F$ is a mad family and $|F|<2^{\aleph_{0}}$. If $B$ is the partition algebra of $F$, then $B$ does not contain the free algebra on $\aleph_{0}$ generators (equivalently, $B$ has no atomless subalgebra).

Proof. If the theorem is false, then there is a sequence of partitioners $\left\langle a_{n}: n \in \omega\right\rangle$ of $F$ such that, for any function $s \in \cup\left\{{ }^{n} 2: n \in \omega\right\}, \cap\left\{a_{n}: s(n)=1\right\} \cap$ $\cap\left\{\omega-a_{n}: s(n)=0\right\}$ is a nontrivial partitioner of $F$. Now if $f \in \in^{\omega} 2$ it is easy to see that there exists an infinite set $a$ such that $\forall n$ if $f(n)=1$ then $a-a_{n}$ is finite and if $f(n)=0$ then $a \cap a_{n}$ is finite. Since $F$ is maximal there is $b \in F$ such that $b \cap a$ is infinite. Since each $a_{n}$ is a partitioner of $F$ we must have $b-a_{n}$ finite when $f(n)=1$ and $b \cap a_{n}$ finite when $f(n)=0$. That is to say, $f$ is canonically obtained from $b$. Since there must be such a $b \in F$ for every $f \in{ }^{\omega} 2$, we must have $|F|=2^{\aleph_{0}}$, a contradiction.

1. Two technical theorems. If $F$ is an almost-disjoint family, then $a$ is a strong partitioner of $F$ iff $a$ is a partitioner of $F$ and both $\{b \in F: b-a$ is finite $\}$ and $\{b \in F: b \cap a$ is finite $\}$ are uncountable.

THEOREM 1.1. Let $I \subseteq P(\omega)$ be an ideal containing at least one infinite set. Then there is an uncountable almost-disjoint family $F \subseteq I$ which is maximal among all almost-disjoint families contained in I and which has no strong partitioners.

Proof. Let $a \in I$ be an infinite set. Let $T=\bigcup\left\{{ }^{n} 2: n \in \omega\right\}$. Then $T$ may be regarded as a tree under inclusion. Without loss of generality we may assume that $I \subseteq P(T)$ and $\{0\} \cup\{s \in T: s(0)=0\} \subseteq a$. 
A set $x \subseteq T$ will be called thin if $\exists f \in^{\omega} 2 \forall n\{s \in x: f \mid n \subseteq s\}$ is cofinite in $x$. Note that $f$ is recoverable from $x$ as the unique path through the subtree $\{t \in T$ : $\exists s \in x t \subseteq s\}$. We denote $f$ by $f_{x}$ in this case. Note that, by König's lemma, every infinite $y \subseteq T$ contains a thin set.

Now let $G$ be an almost-disjoint family of thin sets such that $\{\{f \mid n: n \in \omega\}$ : $\left.f \in^{\omega} 2, f(0)=0\right\} \subseteq G, G \subseteq I$ and $G$ is maximal among all almost-disjoint families contained in $I$.

It is easy to find a bijection $\varphi: G \rightarrow G$ satisfying the following conditions:

(1) $\varphi=\varphi^{-1}$.

(2) If $x \neq y$ and $f_{x}=f_{y}$, then $f_{\varphi(x)} \neq f_{\varphi(y)}$ and either $\varphi(x) \subseteq a$ or $\varphi(y) \subseteq a$.

(3) Either $x \subseteq a$ or $\varphi(x) \subseteq a$.

Let $G=\left\{x_{\alpha}: \alpha<2^{\aleph_{0}}\right\}$. For each $\alpha \leqslant 2^{\aleph_{0}}$ we will obtain a set $X_{\alpha} \subseteq G$ and a bijection $\varphi_{\alpha}$ of $X_{\alpha}$ such that $\left|X_{\alpha}\right| \leqslant|\alpha|+\boldsymbol{\aleph}_{0}$ as follows. Let $\varphi_{0}=0$. Given $\varphi_{\alpha}$, choose $\beta$ minimal such that $x_{\beta} \notin X_{\alpha}$, and let $\gamma$ be minimal such that $x_{\gamma} \subseteq a, x_{\gamma} \notin X_{\alpha} \cup\left\{x_{\beta}\right\}$ and $f_{x_{\gamma}} \notin\left\{f_{y}: y \in X_{\alpha} \cup\left\{x_{\beta}\right\}\right\}$. Let $\varphi_{\alpha+1}=\varphi_{\alpha} \cup\left\{\left(x_{\beta}, x_{\gamma}\right),\left(x_{\gamma}, x_{\beta}\right)\right\}$, and let $X_{\alpha+1}$ $=X_{\alpha} \cup\left\{x_{\beta}, x_{\gamma}\right\}$. Note that $x_{\gamma}$ can always be found since $\left\{f_{x}: x \subseteq a, x \in G, x\right.$ thin $\} \supseteq\left\{\{f \mid n: n \in \omega\}: f \in \in^{\omega} 2, f(0)=0\right\}$, which has cardinality $2^{\aleph_{0}}$. Finally, if $\delta$ is a limit ordinal, let $X_{\delta}=\bigcup\left\{X_{\alpha}: \alpha<\delta\right\}$ and let $\varphi_{\delta}=\bigcup\left\{\varphi_{\alpha}: \alpha<\delta\right\}$. Let $\varphi=\varphi_{2^{\aleph_{0}}}$.

Let $F^{\prime}=\{x \cup \varphi(x): x \in G\}$. Then $F^{\prime}$ is an almost-disjoint family maximal among almost-disjoint families contained in $I$.

Lemma 1.2. If $b$ is a strong partitioner of $F^{\prime}$, then both $\left\{c \in F^{\prime}: c-b\right.$ is finite $\}$ and $\left\{c \in F^{\prime}: c \cap b\right.$ is finite $\}$ have cardinality $2^{\aleph_{0}}$.

Proof. Let us consider $C=\left\{c \in F^{\prime}: c-b\right.$ is finite $\}$. The other case is similar. Let $X=\left\{f_{x}: x \cup \varphi(x) \in C, x \subseteq a\right\}$. Since $C$ is uncountable, so is $X$ by (1)-(3). Let $T^{\prime}=\{s \in T: \exists t \in a \cap b s \subseteq t\}$. Then $T^{\prime}$ is a subtree of $T$ and every element of $X$ is a branch through $T^{\prime}$. Hence the set of branches through $T^{\prime}$ is an uncountable closed subset of ${ }^{\omega} 2$, so there are $2^{\aleph_{0}}$ branches through $T^{\prime}$. But if $f$ is any branch through $T^{\prime}$ then it is easy to find thin $y \subseteq a \cap b$ with $f_{y}=f$. Now $y \in I$ since $a \in I$, so by maximality of $G$ there is $z \in G$ with $z \cap y$ infinite. Hence $z \cap b$ is infinite, so since $b$ is a partitioner of $F^{\prime}, z \cup \varphi(z) \in C$. It follows that $f \in\left\{f_{x}: x \cup \varphi(x) \in C\right\}$, so the latter set has cardinality $2^{\aleph_{0}}$, and $C$ has cardinality $2^{\aleph_{0}}$.

Now we can obtain a bijection $\psi: F^{\prime} \rightarrow F^{\prime}$ such that

(4) $\psi=\psi^{-1}$.

(5) If $b$ is a strong partitioner of $F^{\prime}$ then there is some $x \in F^{\prime}$ such that $x-b$ is finite and $\psi(x) \cap b$ is finite.

(Once again $\psi$ is constructed by an induction of length $2^{\aleph_{0}}$, using Lemma 1.2.) Let $F=\left\{x \cup \psi(x): x \in F^{\prime}\right\}$. Any strong partitioner of $F$ would have to be a strong partitioner of $F^{\prime}$ also, but this is impossible by (5). Thus $F$ is as desired.

Now suppose $U \subseteq P(\omega)$ has the finite intersection property (i.e., every finite intersection of elements of $U$ is infinite).

Note that this conflicts with the usual definition of the finite intersection property; our notion should really be called the strong finite intersection property. 
However, since we have no use for the usual definition we will omit the word "strong" in the rest of the paper.

Let us say that $U$ is extendible iff for any $a \subseteq \omega$, if $U \cup\{a\}$ has the finite intersection property, then there exists an infinite set $b \subseteq \omega$ such that $\forall x \in U \cup\{a\}$, $b-x$ is finite. We say that $U$ is strongly extendible iff for any countable set $X \subseteq P(\omega)$, if $U \cup X$ has the finite intersection property, then there is infinite $b \subseteq \omega$ such that $\forall x \in U \cup X, b-x$ is finite. It is not difficult to find families $U$ which are extendible but not strongly extendible. For example, $\left\{\{(m, n): n>f(m)\}: f \in \in^{\omega} \omega\right\}$ is (isomorphic to) such a family.

THEOREM 1.3. Suppose $B \subseteq P(\omega)$ is a Boolean algebra with the property that every ultrafilter $U$ in $B$ with the finite intersection property (i.e., which contains no finite sets) is strongly extendible. If $I$ is the ideal of finite sets in $B$, then $B / I$ is representable.

Proof. For each ultrafilter $U \subseteq B$ with the finite intersection property, let $I_{U}=\{a \subseteq \omega: \forall b \in U a-b$ is finite $\}$. Then $I_{U}$ is an ideal containing at least one infinite set (by extendibility), so by Theorem 1.1 there is an uncountable family $F_{U} \subseteq I_{U}$ of almost-disjoint sets maximal among all such families and with no strong partitioners. Let $F=\bigcup\left\{F_{U}: U \subseteq B\right.$ an ultrafilter with the finite intersection property . We assert that $F$ is a mad family and $B / I$ is its partition algebra.

Clearly, if $a \in I_{U}, b \in I_{V}$ and $U \neq V$, then $a \cap b$ is finite. Hence $F$ is almostdisjoint. Suppose $a \subseteq \omega$ is infinite. Then there is an ultrafilter $U \subseteq B$ containing no finite sets such that $U \cup\{a\}$ has the finite intersection property (take $U$ to be a maximal filter on $B$ containing the cofinite members of $B$ and such that $U \cup\{a\}$ has the finite intersection property). Hence, since $U$ is extendible, $\exists b \in I_{U} b-a$ is finite. It follows by maximality of $F_{U}$ that $\exists c \in F_{U} b \cap c$ is infinite; hence $a \cap c$ is infinite also and $F$ is maximal.

It is also clear that every element of $B$ is a partitioner of $F$. Moreover, since each $F_{U}$ is infinite, each infinite coinfinite element of $B$ is a nontrivial partitioner of $F$. Let $B^{\prime}$ be the algebra generated by $B \cup F$ and the finite sets, and let $I^{\prime}$ be the ideal in $B^{\prime}$ generated by $F$ and the finite sets. We must show that any nontrivial partitioner differs from an element of $B$ by an element of $I^{\prime}$.

Suppose $a$ is a nontrivial partitioner which does not differ from any element of $B$ by an element of $I^{\prime}$. Then, in particular, $a \in B^{\prime}$, so $\left\{b \in B^{\prime}: b-a\right.$ is finite $\} \cup\{c \in$ $B^{\prime}: c \cap a$ is finite $\} \cup I^{\prime}$ generates a proper ideal $J$ in $B^{\prime}$. Let $U^{\prime}$ be an ultrafilter in $B^{\prime}$ disjoint from $J$, and let $U=U^{\prime} \cap B$.

We claim that $a$ is a strong partitioner of $F_{U}$, contrary to the choice of $F_{U}$. This will complete the proof.

Suppose, for example, that $X=\left\{c \in F_{U}: c-a\right.$ is finite $\}$ is countable or finite. Now $\omega-c \in U^{\prime}$ for each $c \in F$ since $a$ is a partitioner of $F$, so $U \cup\{a\} \cup\{\omega-c$ : $c \in X\}$ has the finite intersection property. Since $U$ is strongly extendible there is infinite $b \subseteq \omega$ such that $\forall x \in U \cup\{a\} \cup\{\omega-c: c \in X\}, b-x$ is finite. Hence $b \in I_{U}$ so $\exists d \in F_{U} b \cap d$ is infinite. It follows that $a \cap d$ is infinite, so $d-a$ is finite since $a$ is a partitioner of $F$. Hence $d \in X$, contradiction. Thus $X$ is uncountable. Similarly we can show that $\left\{c \in F_{U}: c \cap A\right.$ is finite $\}$ is uncountable. 
2. Consequences of Theorem 1.3. We can draw several immediate corollaries from Theorem 1.3.

THEOREM 2.1. Every countable or finite Boolean algebra is representable.

Proof. Every such algebra is embeddable in $P(\omega)$ /Fin, and so is isomorphic to $B /$ Fin for some countable subalgebra $B$ of $P(\omega)$. The strong extendibility condition of Theorem 1.3 is trivially satisfied.

Moreover, certain uncountable algebras are always representable. Let $F C_{\lambda}$ denote the algebra of all finite or cofinite subsets of $\lambda$.

THEOREM 2.2. For all $\lambda \leqslant 2^{\aleph_{0}}, F C_{\lambda}$ is representable.

Proof. As in the previous section, let $T=\cup\left\{{ }^{n} 2: n \in \omega\right\}$. Let $X \subseteq{ }^{\omega} 2$ be such that $|X|=\lambda$, and let $F=\{\{f \mid n: n \in \omega\}: f \in X\}$. Let $B$ be the subalgebra of $P(T)$ generated by $F$, and let $I$ denote the collection of finite sets in $B$. Then clearly $B / I$ is isomorphic to $F C_{\lambda}$. We need check only the strong extendibility condition.

Let $U \subseteq B$ be an ultrafilter with the finite intersection property. If $U$ contains an element of $F$ then $U$ is clearly strongly extendible. Thus we may assume $U$ is the ultrafilter generated by $\{\omega-a: a \in F \cup I\}$. Let $\left\{A_{n}: n \in \omega\right\} \subseteq P(T)$, and suppose $U \cup\left\{A_{n}: n \in \omega\right\}$ has the finite intersection property.

Let $T_{n}=\left\{t \in T: \exists s \in A_{0} \cap \cdots \cap A_{n} t \subseteq s\right\}$, and let $S_{n}=\left\{f \in{ }^{\omega} 2:\{f \mid m: m \in \omega\}\right.$ is a branch through $\left.T_{n}\right\}$.

Case 1. $\cap\left\{S_{n}: n \in \omega\right\}$ is finite. Let $Y=X \cap \cap\left\{S_{n}: n \in \omega\right\}$. Since $U \cup\left\{A_{n}\right.$ : $n \in \omega\}$ has the finite intersection property, for each $n, a_{n}=\left(A_{0} \cap \cdots \cap A_{n}\right)-$ $\cup\{\{f \mid m: m \in \omega\}: f \in Y\}$ is infinite. Hence if $a \subseteq T$ is such that $a-a_{n}$ is finite for all $n$, then $a-b$ is finite for all $b \in U \cup\left\{A_{n}: n \in \omega\right\}$.

Case 2. $\cap\left\{S_{n}: n \in \omega\right\}$ is infinite. It is an easy matter to find distinct $f_{n} \in S_{n}$ so that $\left\{f_{n}: n \in \omega\right\}$ is discrete in ${ }^{\omega} 2$. (Any infinite Hausdorff space has an infinite discrete subspace.) Now fix $\left\langle t_{n}: n \in \omega\right\rangle$ so that $t_{n} \subseteq f_{n}$ and the $t_{n}$ are pairwise incomparable. Finally, choose $s_{n} \in A_{0} \cap \cdots \cap A_{n}$ so that $t_{n} \subseteq s_{n}$ and let $a=\left\{s_{n}\right.$ : $n \in \omega\}$. Now it is easy to check that $a-b$ is finite for all $b \in U \cup\left\{A_{n}: n \in \omega\right\}$.

A closely related atomless algebra is the following. Let $\lambda$ be a cardinal and let $P=\cup\left\{{ }^{n} \lambda: n \in \omega\right\}$. For $p, q \in P$, let $p \leqslant q$ iff $p \supseteq q$. Then $P$ is isomorphic to a dense subset of a complete Boolean algebra $B$. Let $C(\lambda)$ be the subalgebra of $B$ generated by the image of $P$. ("C" stands for "collapse".)

THEOREM 2.3. If $\lambda \leqslant 2^{\aleph_{0}}$ then $C(\lambda)$ is representable.

Proof. The proof is similar to Theorem 2.2, but a little more complicated, so we only sketch it. Let $h: \lambda \rightarrow{ }^{\omega} 2$ be one-to-one, and denote $h(\alpha)$ by $h_{\alpha}$. For each $p \in P$, define an infinite subset $F_{p}$ of $\omega$ and a bijection $G_{p}$ from $F_{p}$ to $T$ by induction as follows. In each case, $G_{p}$ is arbitrarily chosen. Let $F_{0}=\omega$, and let

$$
F_{p^{n}\langle\alpha\rangle}=G_{p}^{-1}\left(\left\{h_{\alpha} \mid n: n \in \omega\right\}\right) .
$$

Let $B$ the subalgebra of $P(\omega)$ generated by $\left\{F_{p}: p \in P\right\} \cup$ Fin. Then it is easily seen that $C(\lambda)$ is isomorphic to $B \mid$ Fin. 
To show that $B$ has the strong extendibility property, let $U$ be a nonprincipal ultrafilter on $B,\left\{A_{n}: n \in \omega\right\} \subseteq P(\omega)$, and suppose $U \cup\left\{A_{n}: n \in \omega\right\}$ has the finite intersection property. The hard case is when there is a $p \in P$ of greatest length such that $F_{p} \in U$. In this case, for each $n \in \omega$ let

$$
\begin{aligned}
& T_{n}=\left\{q \in T:\left(\exists s \in A_{0} \cap \cdots \cap A_{n} \cap F_{p}\right) q \subseteq G_{p}(s)\right\}, \\
& S_{n}=\left\{f \in{ }^{\omega} 2:\{f \mid m: m \in \omega\} \text { is a branch through } T_{n}\right\},
\end{aligned}
$$

and proceed as before.

The interval algebra on the set $\mathbf{R}$ of real numbers, denoted by $I A(\mathbf{R})$, is the subalgebra of $P(\mathbf{R})$ generated by all left-closed, right-open intervals $[a, b)$, where $a$, $b \in \mathbf{R}, a<b$, together with all rays $(-\infty, b)$ where $b \in \mathbf{R}$.

THEOREM 2.4. IA(R) is representable.

Proof. First note that $I A(\mathbf{R})$ is isomorphic to a subalgebra of $P(Q)$, where $Q$ denotes the rationals, by the mapping which carries $a \in I A(\mathbf{R})$ to $a \cap Q$. Then observe that every ultrafilter in $I A(\mathbf{R})$ is countably generated. The result now follows by Theorem 1.3.

It is worth pointing out that if $B$ is an algebra satisfying the hypotheses of Theorem 1.3, then any subalgebra of $B$ also satisfies the hypotheses. We do not know, however, whether a subalgebra of a representable algebra must necessarily be representable.

Following van Douwen, we let $p$ denote the smallest cardinal number such that for any family $F \subseteq P(\omega)$, if $|F|<p$ and $F$ has the finite intersection property, then there is infinite $a \subseteq \omega$ such that $a-b$ is finite for all $b \in F$. It is well known, for example, that Martin's Axiom implies that $p=2^{\aleph_{0}}$, whereas the converse is false (see [6]). Rothberger [8] was the first to consider the proposition $p=2^{\aleph_{0}}$.

The following theorem is an immediate consequence of Theorem 1.3.

THEOREM 2.5. If $B$ is a subalgebra of $P(\omega) /$ Fin and $|B|<p$, then $B$ is representable.

We can even do a little better.

THEOREM 2.6. If $B$ is a Boolean algebra and $|B|<p$, then $B$ is embeddable in $P(\omega) /$ Fin, so $B$ is representable.

Proof. It seems to be part of the folklore that Martin's Axiom implies that every Boolean algebra of cardinality $<2^{\aleph_{0}}$ is embeddable in $P(\omega) /$ Fin. The argument given here is a mild variation of the usual one, making use of a result of Murray Bell.

Fix $B$, and let $P$ be the following partial ordering. Let $p \in P$ iff $p$ is finite, domain $(p) \subseteq B$ and range $(p) \subseteq^{n} 2$ for some $n=n(p) \in \omega$. Let $p \leqslant q$ iff

(1) domain $(p) \supseteq \operatorname{domain}(q)$,

(2) $\forall b \in \operatorname{domain}(q), p(b) \supseteq q(b)$,

(3) $\forall a, b, c \in \operatorname{domain}(q)$ if $a \wedge b=c$ then $\forall m \in \operatorname{domain}(p)$ if $m \geqslant n(q)$ then $p(c)(m)=1$ iff $p(a)(m)=p(b)(m)=1$,

(4) $\forall a, b \in \operatorname{domain}(q)$ if $a=\bar{b}$ then $\forall m \in \operatorname{domain}(p)$ if $m \geqslant n(q)$ then $p(a)(m)$ $=1$ iff $p(b)(m)=0$. 
For $a, b \in B, a \neq b$, and $n \in \omega$ let $D(a, b, n)=\{p \in P: a, b \in \operatorname{domain}(p), n<$ $n(p)$ and $\exists m \geqslant n p(a)(m) \neq p(b)(m)\}$. It should be clear that each $D(a, b, n)$ is dense in $P$. If $G \subseteq P$ is a pairwise compatible set and $D(a, b, n) \cap G \neq 0$ for all $a, b$ and $n$, then it is easy to see that $B$ may be embedded into $P(\omega) /$ Fin by mapping $a$ to the equivalence class of $\{n: \exists p \in G p(a)(n)=1\}$.

Recall that a partial ordering $Q$ is $\sigma$-centered if there exists $f: Q \rightarrow \omega$ such that $\forall n$ every finite subset of $f^{-1}\{n\}$ has a lower bound. Bell [1] showed that MA $\mathbf{A}_{\kappa}$ holds for $\sigma$-centered partial orderings whenever $\kappa<p$, so we need only check that $P$ is $\sigma$-centered.

Define another partial ordering on $P$ by letting $p \leqslant^{\prime} q$ iff $p \supseteq q$. Then it is clear that if $F$ is a finite subset of $P$ with a lower bound relative to $\leqslant^{\prime}$, then $F$ has a lower bound relative to $\leqslant$. (Note that $n(p)=n(q)$ for all $p, q \in F$.) But $\left(P, \leqslant^{\prime}\right)$ is essentially the Cohen ordering for adding $|B|$ subsets of $\omega$, and it is well known that this ordering is $\sigma$-centered, since $|B| \leqslant 2^{\kappa_{0}}$. (This can be seen as follows: The Stone space $X$ of the Cohen algebra is the product of $2^{\aleph_{0}}$ copies of the two-element discrete space, hence is separable. Let $\left\{x_{n}: n \in \omega\right\}$ be dense in $X$. Now define $f: P \rightarrow \omega$ so that if $U_{p}$ is the open set in $X$ determined by $p$, then $x_{f(p)} \in U_{p}$.) Hence $(P, \leqslant)$ is also $\sigma$-centered and the proof is complete.

Unfortunately, it follows from a result of Kunen [5] that Theorem 2.6 does not extend to Boolean algebras of cardinality $2^{\aleph_{0}}$, even assuming Martin's Axiom, for such algebras need not be embeddable in $P(\omega) /$ Fin. Nevertheless it is conceivable that these algebras are still representable, but we have not been able to prove it.

3. All algebras may be representable. In Theorem 2.1 we saw that every countable Boolean algebra is representable. It is well known (see [2, Theorem 14.12]) that every Boolean algebra of cardinality $\aleph_{1}$ is embeddable in $P(\omega)$ /Fin, so it is natural to ask whether every such algebra is representable. We have not been able to answer this question in general, but here we give an affirmative answer assuming the continuum hypothesis.

TheOrem 3.1. Assume CH. Then every Boolean algebra of cardinality $\leqslant 2^{\aleph_{0}}$ is representable.

Proof. Let $B$ be a Boolean algebra of cardinality $2^{\aleph_{0}}=\aleph_{1}$, and let $\left\langle b_{\alpha}: \alpha<\omega_{1}\right\rangle$ be a sequence of generators for $B$ such that, if $B_{\alpha}$ is the subalgebra generated by $\left\{b_{\beta}\right.$ : $\beta<\alpha\}$, then $b_{\alpha} \notin B_{\alpha}$ for all $\alpha$. Let $\left\langle z_{\alpha}: \alpha<\omega_{1}\right\rangle$ enumerate the infinite subsets of $\omega$. By induction on $\alpha<\omega_{1}$ we will construct $C_{\alpha}, F_{\alpha}$ and $f_{\alpha}$ such that:

(1) $F_{\alpha}$ is a countable almost-disjoint family.

(2) $C_{\alpha}$ is a countable subalgebra of $P(\omega)$ containing $F_{\alpha}$ and the finite sets, and consisting exclusively of partitioners of $F_{\alpha}$; moreover $\forall a \in C_{\alpha}$ if $a$ is infinite then $\exists b \in F_{\alpha} b-a$ is finite.

(3) $f_{\alpha}: C_{\alpha} \rightarrow B_{\alpha}$ is a surjective homomorphism, and the kernel of $f_{\alpha}$ is the ideal $I_{\alpha}$ generated by $F_{\alpha}$ and the finite sets.

(4) If $\alpha<\beta$ then $C_{\alpha} \subseteq C_{\beta}, F_{\alpha} \subseteq F_{\beta}$ and $f_{\alpha} \subseteq f_{\beta}$.

(5) $\forall \alpha$ either $z_{\alpha} \in C_{\alpha+1}$ or else $z_{\alpha}$ is not a partitioner of $F_{\alpha+1}$. 
Once this construction has been accomplished, let $F=\bigcup\left\{F_{\alpha}: \alpha<\omega_{1}\right\}, C=$ $\bigcup\left\{C_{\alpha}: \alpha<\omega_{1}\right\}, I=\bigcup\left\{I_{\alpha}: \alpha<\omega_{1}\right\}, f=\bigcup\left\{f_{\alpha}: \alpha<\omega_{1}\right\}$. Then by (2) and (5) $F$ is a maximal almost-disjoint family and $C$ is the set of partitioners of $F$; by (3) and (4) $f$ induces an isomorphism of $C / I$ with $B$, as desired.

Now we treat the construction. By Theorem 2.1, we may start the construction at stage $\alpha=\omega$. (This may involve redefining $z_{n}$ for $n<\omega$.) If $\alpha$ is a limit ordinal, let $C_{\alpha}=\bigcup\left\{C_{\beta}: \beta<\alpha\right\}, F_{\alpha}=\bigcup\left\{F_{\beta}: \beta<\alpha\right\}, f_{\alpha}=\bigcup\left\{f_{\beta}: \beta<\alpha\right\}$. Clearly (1)-(5) are satisfied.

Finally, suppose $C_{\alpha}, F_{\alpha}$ and $f_{\alpha}$ have been obtained. We will produce $C_{\alpha+1}, F_{\alpha+1}$ and $f_{\alpha+1}$ in two steps. First we define $C_{\alpha}^{\prime}, F_{\alpha+1}$ and $f_{\alpha}^{\prime}$. If $z_{\alpha} \in C_{\alpha}$ or $z_{\alpha}$ is not a partitioner of $F_{\alpha}$, then let $F_{\alpha+1}=F_{\alpha}, C_{\alpha}^{\prime}=C_{\alpha}, f_{\alpha}^{\prime}=f_{\alpha}$. Otherwise let $I^{\prime}$ be the ideal in $C_{\alpha}$ generated by $I_{\alpha}$ together with $\left\{a \in C_{\alpha}: a \cap z_{\alpha}=0\right\} \cup\left\{a \in C_{\alpha}: a-z_{\alpha}=0\right\}$. Since $z_{\alpha} \notin C_{\alpha}$ but $z_{\alpha}$ is a partitioner of $F_{\alpha}$, it is easy to see that $I^{\prime}$ is proper.

Let $U$ be an ultrafilter in $C_{\alpha}$ disjoint from $I^{\prime}$. Note that $\forall a \in U$ both $a \cap z_{\alpha}$ and $a-z_{\alpha}$ are infinite. Now let $y_{1}$ and $y_{2}$ be infinite sets such that $\forall a \in U, y_{1}-\left(a \cap z_{\alpha}\right)$ and $y_{2}-\left(a-z_{\alpha}\right)$ are finite. Let $F_{\alpha+1}=F_{\alpha} \cup\left\{y_{1} \cup y_{2}\right\}$ and let $C_{\alpha}^{\prime}$ be the algebra generated by $C_{\alpha} \cup\left\{y_{1} \cup y_{2}\right\}$. Note that $F_{\alpha+1}$ is an almost-disjoint family, $C_{\alpha}^{\prime} \supseteq F_{\alpha+1}$ $\cup$ Fin, $C_{\alpha}^{\prime}$ consists exclusively of partitioners of $F_{\alpha+1}$, and $\forall a \in C_{\alpha}^{\prime}$ if $a$ is infinite then $\exists b \in F_{\alpha+1} b-a$ is finite; and $z_{\alpha}$ is not a partitioner of $F_{\alpha+1}$. If $y=y_{1} \cup y_{2}$, then every element of $C_{\alpha}^{\prime}$ has the form $(a \cap y) \cup(b-y)$ for $a, b \in C_{\alpha}$. Let $f_{\alpha}^{\prime}((a \cap y) \cup(b-y))=f_{\alpha}(b)$. We must check that $f_{\alpha}^{\prime}$ is well defined. If $\left(a_{1} \cap y\right) \cup$ $\left(b_{1}-y\right)=\left(a_{2} \cap y\right) \cup\left(b_{2}-y\right)$, then the symmetric difference $b_{1}+b_{2} \subseteq y$. Since $b_{1}+b_{2} \in C_{\alpha}$, but $y \notin C_{\alpha}$ (as is easily seen), we must have by (2) that $b_{1}+b_{2}$ is finite. But then $f_{\alpha}\left(b_{1}\right)=f_{\alpha}\left(b_{2}\right)$ so $f_{\alpha}^{\prime}$ is well defined; clearly it is a homomorphism from $C_{\alpha}^{\prime}$ into $B_{\alpha}$.

Now, given $C_{\alpha}^{\prime}, F_{\alpha+1}$ and $f_{\alpha}^{\prime}$, we construct $C_{\alpha+1}$ and $f_{\alpha+1}$. Let $P=\{(a, b)$ : $\left.a, b \in C_{\alpha}^{\prime}, a \cap b=0, f_{\alpha}^{\prime}(a) \leqslant b_{\alpha}, f_{\alpha}^{\prime}(b) \leqslant \bar{b}_{\alpha}\right\}$. Since $b_{\alpha} \notin B_{\alpha}$, we have $\omega-(a \cup b)$ infinite for all $(a, b) \in P$. Let $P$ be partially ordered by letting $(a, b) \leqslant(c, d)$ iff $c \subseteq a$ and $d \subseteq b$. We consider the following subsets of $P$, all of which are easily seen to be dense:

Let $I_{\alpha}^{\prime}$ be the ideal in $C_{\alpha}^{\prime}$ generated by $I_{\alpha} \cup F_{\alpha+1}$; it is easily seen that $I_{\alpha}^{\prime}$ is the kernel of $f_{\alpha}$. If $a \in C_{\alpha}^{\prime}$ and $f_{\alpha}^{\prime}(a) \leqslant b_{\alpha}$, then let $D(a)=\left\{\left(a^{\prime}, b^{\prime}\right) \in P: a-a^{\prime} \in I_{\alpha}^{\prime}\right\}$. If $f_{\alpha}^{\prime}(a) \leqslant \bar{b}_{\alpha}$ then let $D(a)=\left\{\left(a^{\prime}, b^{\prime}\right) \in P: a-b^{\prime} \in I_{\alpha}^{\prime}\right\}$. If $f_{\alpha}^{\prime}(a) \wedge b_{\alpha}$ and $f_{\alpha}^{\prime}(a)-b_{\alpha}$ are both nonzero, then let $D(a)=\left\{\left(a^{\prime}, b^{\prime}\right) \in P: \exists c, d \in F_{\alpha+1} c-\left(a \cap b^{\prime}\right)\right.$ are both finite $\}$.

For $a, b \in C_{\alpha}^{\prime}$, let $D_{1}(a, b)=\left\{\left(a^{\prime}, b^{\prime}\right) \in P\right.$ : either $a^{\prime} \cap a \neq a^{\prime} \cap b$ or else $a-b^{\prime}$ $\left.=b-b^{\prime}\right\}$, and let $D_{2}(a, b)=\left\{\left(a^{\prime}, b^{\prime}\right) \in P\right.$ : either $b^{\prime} \cap a \neq b^{\prime} \cap b$ or else $a-a^{\prime}=$ $\left.b-a^{\prime}\right\}$. For $a \in F_{\alpha+1}$ let $E(a)=\left\{\left(a^{\prime}, b^{\prime}\right) \in P: a-\left(a^{\prime} \cup b^{\prime}\right)\right.$ is finite $\}$, and for $n \in \omega$ let $D_{n}=\left\{\left(a^{\prime}, b^{\prime}\right) \in P: n \in a^{\prime} \cup b^{\prime}\right\}$.

Let $G$ be generic with respect to all these (countably many) dense sets, and let $a_{\alpha}=\cup\{a: \exists b(a, b) \in G\}$.

Let $C_{\alpha+1}$ be the algebra generated by $C_{\alpha}^{\prime} \cup\left\{a_{\alpha}\right\}$. Note that since $G \cap E(a) \neq 0$ for all $a \in F_{\alpha+1}, a_{\alpha}$ is a partitioner of $F_{\alpha+1}$; in fact, if $\left(a^{\prime}, b^{\prime}\right) \in G \cap E(a)$ and $a \cap a^{\prime}$ is finite, it is easily seen that $a \cap a_{\alpha}=a \cap a^{\prime}$. Hence $C_{\alpha+1}$ consists of partitioners of $F_{\alpha+1}$. 
Every element of $C_{\alpha+1}$ has the form $\left(a \cap a_{\alpha}\right) \cup\left(b-a_{\alpha}\right)$ for $a, b \in C_{\alpha}^{\prime}$, and every element of $B_{\alpha+1}$ has the form $\left(a \wedge b_{\alpha}\right) \vee\left(b-b_{\alpha}\right)$ for $a, b \in B_{\alpha}$. Define $f_{\alpha+1}$ : $C_{\alpha+1} \rightarrow B_{\alpha+1}$ by

$$
f_{\alpha+1}\left(\left(a \cap a_{\alpha}\right) \cup\left(b-a_{\alpha}\right)\right)=\left(f_{\alpha}^{\prime}(a) \wedge b_{\alpha}\right) \vee\left(f_{\alpha}^{\prime}(b)-b_{\alpha}\right) .
$$

LEMMA 3.2. $f_{\alpha+1}$ is well defined.

PROOF. If $\left(a_{1} \cap a_{\alpha}\right) \cup\left(b_{1}-a_{\alpha}\right)=\left(a_{2} \cap a_{\alpha}\right) \cup\left(b_{2}-a_{\alpha}\right)$, then $a_{1} \cap a_{\alpha}=a_{2} \cap$ $a_{\alpha}$ and $\left(b_{1}-a_{\alpha}\right)=\left(b_{2}-a_{\alpha}\right)$. Let $(a, b) \in G \cap D_{1}\left(a_{1}, a_{2}\right)$. Then we must have had $a_{1}-b=a_{2}-b$, while $f_{\alpha}^{\prime}(b) \leqslant \bar{b}_{\alpha}$. Thus

$$
\begin{aligned}
f_{\alpha}^{\prime}\left(a_{1}\right) \wedge b_{\alpha} & =f_{\alpha}^{\prime}\left(\left(a_{1}-b\right) \cup b\right) \wedge b_{\alpha}=\left[f_{\alpha}^{\prime}\left(a_{1}-b\right) \vee f_{\alpha}^{\prime}(b)\right] \wedge b_{\alpha} \\
& =f_{\alpha}^{\prime}\left(a_{1}-b\right) \wedge b_{\alpha}=f_{\alpha}^{\prime}\left(a_{2}-b\right) \wedge b_{\alpha}=f_{\alpha}^{\prime}\left(a_{2}\right) \wedge b_{\alpha} .
\end{aligned}
$$

Similarly, using $D_{2}\left(b_{1}, b_{2}\right)$, we can show $f_{\alpha}^{\prime}\left(b_{1}\right)-b_{\alpha}=f_{\alpha}^{\prime}\left(b_{2}\right)-b_{\alpha}$.

LEMMA 3.3. $f_{\alpha+1}$ is surjective.

Proof. This is obvious, since $f_{\alpha}^{\prime}$ is surjective.

LEMMA 3.4. $f_{\alpha+1}$ is a homomorphism.

Proof. This is routine.

LEMMA 3.5. The kernel of $f_{\alpha+1}$ is $I_{\alpha+1}$, the ideal generated by $F_{\alpha+1}$ and the finite sets.

Proof. It is clear that $f_{\alpha+1} \supseteq f_{\alpha}^{\prime}$, and $F_{\alpha+1}$ and the finite sets are contained in the kernel of $f_{\alpha}^{\prime}$, so $I_{\alpha+1}$ is contained in the kernel of $f_{\alpha+1}$. Suppose

$$
f_{\alpha+1}\left(\left(a \cap a_{\alpha}\right) \cup\left(b-a_{\alpha}\right)\right)=0 .
$$

Then $f_{\alpha}^{\prime}(a) \wedge b_{\alpha}=0$ and $f_{\alpha}^{\prime}(b)-b_{\alpha}=0$. We will show that $a \cap a_{\alpha} \in I_{\alpha+1}$; the proof that $b-a_{\alpha} \in I_{\alpha+1}$ is similar. But $f_{\alpha}^{\prime}(a) \wedge b_{\alpha}=0$ implies $f_{\alpha}^{\prime}(a) \leqslant \bar{b}_{\alpha}$. If $\left(a^{\prime}, b^{\prime}\right)$ $\in G \cap D(a)$, then $a-b^{\prime} \in I_{\alpha}^{\prime}$. Hence $a \cap a_{\alpha}=\left(a-b^{\prime}\right) \cap a_{\alpha} \subseteq a-b^{\prime} \in I_{\alpha}^{\prime}$ so $a \cap a_{\alpha} \in I_{\alpha+1}$ and we are done.

Lemma 3.6. If $a \in C_{\alpha+1}$ is infinite then $\exists b \in F_{\alpha+1} b-a$ is finite.

Proof. We assume $a=a^{\prime} \cap a_{\alpha}$ for $a^{\prime} \in C_{\alpha}^{\prime}$; the case $a=a^{\prime}-a_{\alpha}$ is similar. If $a^{\prime} \in F_{\alpha+1}$ then since $E\left(a^{\prime}\right) \cap G \neq 0$ we must have $a^{\prime}-a_{\alpha}=a^{\prime}-a$ finite. Thus we may assume $a^{\prime} \notin I_{\alpha}^{\prime}$. If $f_{\alpha}^{\prime}\left(a^{\prime}\right) \leqslant b_{\alpha}$ then since $D\left(a^{\prime}\right) \cap G \neq 0$ we are done since $C_{\alpha}^{\prime}$ has the property of this lemma. If $f_{\alpha}^{\prime}\left(a^{\prime}\right) \leqslant \bar{b}_{\alpha}$ then since $D\left(a^{\prime}\right) \cap G \neq 0$ we would have $a^{\prime} \cap a_{\alpha}=b \cap a_{\alpha}$ for some $b \in I_{\alpha}^{\prime}$, and this case has already been treated. In the only remaining case there is still no difficulty, since again $D\left(a^{\prime}\right) \cap G \neq 0$.

This completes the proof of Theorem 3.1.

4. Some algebras may not be representable. In contrast to the result of $\S 3$, here we show that the existence of nonrepresentable algebras of cardinality $\leqslant 2^{\kappa_{0}}$ is relatively consistent with the axioms of set theory.

If $\kappa$ is a cardinal, let $P(\kappa, \omega)$ denote the set of all finite functions $p$ such that domain $(p) \subseteq \kappa$ and range $(p) \subseteq 2$, ordered by reverse inclusion. Then $P(\kappa, \omega)$ is the usual ordering for adding $\kappa$ Cohen-generic subsets of $\omega$. 
Theorem 4.1. Assume CH. Let $\kappa \geqslant \boldsymbol{\aleph}_{2}$ be a cardinal, and let $P=P(\kappa, \omega)$. Then $\mathbb{}_{P}$ "If $B$ has a subalgebra isomorphic to the free algebra on $\aleph_{2}$ generators, then $B$ is not representable". Hence $\mathbb{}_{P}$ "No infinite complete Boolean algebra is representable".

Proof. Suppose, by way of contradiction, that the theorem is false. We may assume that $G$ is $P$-generic over $V$, and in $V[G]$ there is a mad family $F$ and a sequence $\left\langle a_{\xi}: \xi<\omega_{2}\right\rangle$ of subsets of $\omega$ such that $\forall p \in P\left(\omega_{2}, \omega\right), \cap\left\{a_{\xi}: p(\xi)=1\right\}$ $\cap \cap\left\{\omega-a_{\xi}: p(\xi)=0\right\}$ is a nontrivial partitioner of $F$.

It follows immediately that if $f \in V[G]$ is a countable function with domain $(f) \subseteq$ $\omega_{2}$, range $(f) \subseteq 2$, then $\exists a \in F \forall \xi \in \operatorname{domain}(f) a-a_{\xi}$ is finite if $f(\xi)=1$ and $a \cap a_{\xi}$ is finite if $f(\xi)=0$. For there must exist infinite $b$ such that $b-a_{\xi}$ is finite if $f(\xi)=1$ and $b \cap a_{\xi}$ is finite if $f(\xi)=0$, and by maximality of $F$ there is $a \in F$ with $a \cap b$ infinite; but now the conclusion follows by the assumption of the preceding paragraph.

By the homogeneity of $P$, the assertion of the first paragraph is forced with respect to $P$. From now on we work in $V$. Let $\dot{F}$ and $\left\langle\dot{a}_{\xi}: \xi<\omega_{2}\right\rangle$ be terms denoting $F$ and $\left\langle a_{\xi}: \xi<\omega_{2}\right\rangle$.

It is well known that if $\varphi$ is any sentence of the language of forcing then there is a countable set $X \subseteq \kappa$ such that

$$
\forall p \in P p \Vdash \varphi \quad \text { iff } p \mid X \Vdash \varphi .
$$

It follows that for each $\xi<\omega_{2}$ there is countable $X_{\xi} \subseteq \kappa$ such that $\forall p \in P \forall n \in \omega$ :

$$
p \Vdash n \in \dot{a}_{\xi} \Leftrightarrow p \mid X_{\xi} \Vdash n \in \dot{a}_{\xi}
$$

and

$$
p \Vdash n \notin \dot{a}_{\xi} \Leftrightarrow p \mid X_{\xi} \Vdash n \notin \dot{a}_{\xi} .
$$

By $\mathrm{CH}$, we know that there exists $Z \subseteq \omega_{2}$ such that $|Z|=\boldsymbol{\aleph}_{2}$ and $\left\{X_{\xi}: \xi \in Z\right\}$ is a $\Delta$-system; hence we may assume that $\left\{X_{\xi}: \xi<\omega_{2}\right\}$ is a $\Delta$-system with kernel $\Delta$ (i.e., $X_{\xi} \cap X_{\eta}=\Delta$ whenever $\xi<\eta<\omega_{2}$ ).

Let $P \mid X_{\xi}=\left\{p \mid X_{\xi}: p \in P\right\}$. Note that if $f$ is a bijection between $X_{\xi}$ and $X_{\eta}$ then $f$ naturally lifts to an isomorphism $f^{*}$ between $P \mid X_{\xi}$ and $P \mid X_{\eta}$. Just define $f^{*}$ so that $f^{*}(p)(f(\alpha))=p(\alpha)$. Let us say that $\dot{a}_{\xi}$ and $\dot{a}_{\eta}$ are isomorphic if there is a bijection $f$ : $X_{\xi} \rightarrow X_{\eta}$ such that $\forall p \in P \mid X_{\xi} \forall n \in \omega$,

$$
p \Vdash n \in \dot{a}_{\xi} \Leftrightarrow f^{*}(p) \Vdash n \in \dot{a}_{\eta}
$$

and

$$
p \Vdash n \notin \dot{a}_{\xi} \Leftrightarrow f^{*}(p) \Vdash n \notin \dot{a}_{\eta} .
$$

It is easy to see that there are at most $2^{\aleph_{0}}$ isomorphism types among the $\dot{a}_{\xi}$ so, by $\mathrm{CH}$, we may assume without loss of generality that all the $\dot{a}_{\xi}$ are isomorphic.

Finally, let us say that $\dot{a}_{\xi}$ and $\dot{a}_{\eta}$ are $\Delta$-isomorphic if they are isomorphic via a bijection $f: X_{\xi} \rightarrow X_{\eta}$ which is the identity on $\Delta$. Again it is clear that there are at most $2^{\aleph_{0}} \Delta$-isomorphism types, so we may assume that all the $\dot{a}_{\xi}$ are $\Delta$-isomorphic. 
Fix $\delta<\kappa$ so that $\{\delta+n: n \in \omega\} \cap \cup\left\{X_{m}: m \in \omega\right\}=0$. Now if $\dot{G}$ denotes the generic set $G$ we have, by our observation above,

$$
\begin{aligned}
& \Vdash_{P} \exists a \in \dot{F} \forall n \in \omega a-\dot{a}_{n} \text { is finite if } \cup \dot{G}(\delta+n)=1 \text { and } a \cap \dot{a}_{n} \\
& \text { is finite if } \cup \dot{G}(\delta+n)=0 .
\end{aligned}
$$

Let $\dot{a}$ be a term so that the statement above is forced when $a$ is replaced by $\dot{a}$.

Let $Y \subseteq \kappa$ be a countable set related to $\dot{a}$ as $X_{\xi}$ is related to $\dot{a}_{\xi}$ in (1) and (2). Finally, choose $\alpha$ so large that

$$
\left(X_{\alpha}-\Delta\right) \cap\left(Y \cup\{\delta+n: n \in \omega\} \cup \cup\left\{X_{m}: m \in \omega\right\}\right)=0 .
$$

We assert that

$$
\Vdash_{P} \dot{a}-\dot{a}_{\alpha} \text { and } \dot{a} \cap \dot{a}_{\alpha} \text { are both infinite, }
$$

which contradicts our original assumption. This will complete the proof.

We will show that $\forall p \in P \forall n \in \omega \exists q \leqslant p \exists m>n q \Vdash m \in \dot{a} \cap \dot{a}_{\alpha}$. The proof for $\dot{a}-\dot{a}_{\alpha}$ is similar. Fix $p \in P$. Since $p$ is finite there is $k \in \omega$ large enough so that $\delta+k \notin \operatorname{domain}(p)$ and $X_{k} \cap \operatorname{domain}(p) \subseteq \Delta$. Let $f: X_{\alpha} \rightarrow X_{k}$ be a $\Delta$-isomorphism. By assumption on $k f^{*}\left(p \mid X_{\alpha}\right)$ is compatible with $p$. Let $q=f^{*}\left(p \mid X_{\alpha}\right) \cup p \cup$ $\{(\boldsymbol{\delta}+k, 1)\}$.

Now $q \Vdash \dot{a}-\dot{a}_{k}$ is finite, so $q \Vdash \dot{a} \cap \dot{a}_{k}$ is infinite. Hence there is $r \leqslant q$ and $m \geqslant n$ such that $r \Vdash m \in \dot{a} \cap \dot{a}_{k}$. Moreover, $r \mid Y \cup X_{k} \Vdash m \in \dot{a} \cap \dot{a}_{k}$ by (1) for $Y$ and $X_{k}$, so we may assume that $r=q \cup r \mid Y \cup X_{k}$.

But this means $r\left|X_{\alpha}=p\right| X_{\alpha}$ by choice of $\alpha$, so $s=r \cup f^{*-1}\left(r \mid X_{k}\right)$ is a condition, and clearly $s \Vdash m \in \dot{a} \cap \dot{a}_{\alpha}\left(s \Vdash m \in \dot{a}\right.$ since $s \leqslant r$, and $s \Vdash m \in \dot{a}_{\alpha}$ since $r \mid X_{k} \Vdash m$ $\in \dot{a}_{k}, f^{*-1}\left(r \mid X_{k}\right) \leqslant s$, and $f^{*}$ is a $\Delta$-isomorphism).

This completes the proof of the first part of the theorem.

For the last assertion, simply note that any infinite Boolean algebra contains a disjoint family $\left\{b_{n}: n \in \omega\right\}$ of nonzero elements. If $A$ is a family of independent subsets of $\omega$ and $|A|=2^{\aleph_{0}}$, then $\left\{\bigvee\left\{b_{n}: n \in a\right\}: a \in A\right\}$ generates a free algebra of cardinality $2^{\aleph_{0}}$. Since $2^{\aleph_{0}} \geqslant \aleph_{2}$ in the generic extension, it follows that any infinite complete Boolean algebra has a subalgebra isomorphic to the free algebra on $\boldsymbol{\aleph}_{2}$ generators.

One of the referees has remarked that a similar argument may be used to conclude that

$\Vdash_{P}$ "If $B$ has a chain of order type $\omega_{2}$, then $B$ is not representable".

5. Open problems. We conclude with a list of open problems.

Problem 1. If $B$ is representable, must every subalgebra of $B$ be representable?

Problem 2. If $B$ is representable, must every homomorphic image of $B$ be representable?

Problem 3. Must every representable algebra be embeddable in $P(\omega) /$ Fin?

Problem 4. Is it provable in ZFC that every Boolean algebra of cardinality $\boldsymbol{N}_{1}$ is representable?

Problem 5. Is it provable in ZFC that the free algebra on $\boldsymbol{\aleph}_{1}$ generators is representable? 
Problem 6. In Theorem 1.3 can strong extendibility be replaced by extendibility?

Problem 7. Is it consistent with $-\mathrm{CH}$ that every Boolean algebra of cardinality $\leqslant 2^{\aleph_{0}}$ be representable?

There are also some less specific problems.

Problem 8. Characterize the Boolean algebras which are always (i.e., in ZFC) representable.

Problem 9. Characterize the algebras representable when "almost-disjoint" is defined with respect to an ideal other than Fin.

Problem 10. Generalize this discussion to cardinals above $\aleph_{0}$.

A Boolean algebra is superatomic if it has no atomless subalgebras. Let us call a mad family $F$ small if $|F|<2^{\aleph_{0}}$. The methods of Theorems $0.1,1.3,2.1$ and 2.2 may be used to show that if there exists a small mad family, then every countable superatomic Boolean algebra together with certain of the $F C_{\lambda}, \boldsymbol{\aleph}_{1} \leqslant \lambda<2^{\aleph_{0}}$, are representable by small mad families.

Problem 11. Suppose that there is a mad family $F$ with $|F|=\lambda<2^{\aleph_{0}}$. Characterize the superatomic Boolean algebras which are representable by mad families of cardinality $\lambda$.

\section{REFERENCES}

1. M. Bell, On the combinatorial principle $P(c)$, Fund. Math. 114 (1981), 149-157.

2. W. W. Comfort and S. Negrepontis, The theory of ultrafilters, Springer-Verlag, Berlin and New York, 1974.

3. S. Hechler, Classifying almost-disjoint families with applications to $\beta N-N$, Israel J. Math. 10 (1971), 413-432.

4. Short complete nested sequences in $\beta N-N$ and small maximal almost-disjoint families, General Topology Appl. 2 (1972), 139-149.

5. K. Kunen, mimeographed notes.

6. K. Kunen and F. Tall, Between Martin's Axiom and Souslin's Hypothesis, Fund. Math. 102 (1979), 173-181.

7. A. R. D. Mathias, Happy families, Ann. Math. Logic 12 (1977), 59-111.

8. F. Rothberger, On some problems of Hausdorff and of Sierpiński, Fund. Math. 35 (1948), 29-46.

9. M. Weese, Mad families and ultrafilters, Proc. Amer. Math. Soc. 80 (1980), 475-477.

Department of Mathematics, Dartmouth College, Hanover, New Hampshire 03755

Department of Mathematics, Humboldt-Universität zu Berlin, Berlin, DDR 